\title{
MODELING OF THERMOHYDRAULIC PROCESES OF HEAT EXCHANGE EQUIPMENT
}

\author{
Karina Savina ${ }^{1}$, Alexey Abramovskih ${ }^{1}$, Vladimir Haymin ${ }^{1}$, and Sergey Lavrinenko ${ }^{1, *}$ \\ ${ }^{1}$ National Research Tomsk Polytechnic University, 634050 Tomsk, Russia
}

\begin{abstract}
You should leave $8 \mathrm{~mm}$ of space above the abstract and $10 \mathrm{~mm}$ after the abstract. The heading Abstract should be typed in bold 9-point Arial. The body of the abstract should be typed in normal 9-point Times in a single paragraph, immediately following the heading. The text should be set to 1 line spacing. The abstract should be centred across the page, indented $17 \mathrm{~mm}$ from the left and right page margins and justified. It should not normally exceed 200 words.
\end{abstract}

\section{Introduction}

As it is known [1], the development of heat exchangers for mobile power stations and modular units is an actual problem of modern heat engineering, housing, communal service and many other coupled industries. Special attention is given to projects of energy efficient equipment in the regions with an autonomous power supply system. It should be noted, that in the Russia a project to create mobile floating nuclear power plants of small power and reduced-enrichment uranium fuel possibility [2] was propsed by State Corporation for Atomic Energy "Rosatom" [3-5].

Operating experience of block-modular facilities produce thermal and electrical energy, it leads to the conclusion that the technical and economic indicators depends on operating conditions.

The research results showed that it is necessary to carry out modeling, taking into account the specific conditions of the operation of power unit in order to determine the efficiency of heat exchange equipment.

\section{Numerical analysis}

Based on the foregoing numerical analysis of the effectiveness of the degasser was carried out, using the FLOW SIMULATION Solid Works package block for calculating. This unit makes possible to perform 3-dimensional finite element stationary and non-stationary calculations for real geometry considering the nonlinear properties of solids and fluids. The accepted calculation model considers both mechanisms of internal heat transfer and heat transfer from the degasser case to the environment.

\footnotetext{
*Corresponding author: serg86@tpu.ru
} 
The software package FLOW SIMULATION provides the possibility to simulate a convective fluid flow in closed and open areas with various fluids and pressures.

The complex tasks related to the issues of hydrodynamics and heat exchange in the SolidWorks Flow Simulation is solved by a system of differential equations of motion, continuity, energy, the thermal conductivity in the channel walls $[6,7]$.

The main stages of the development of computer models in SolidWorks Flow Simulation are [1]:

a) the creation of a 3D model;

b) the construction of a grid computational domain;

c) the imposition of boundary conditions;

d) the calculation and visualization of the derived temperature field, pressure, etc.

\section{Computational models}

As the initial data a 3-D model of the degasser's structure was used. In the first stage, the area of the degasser with the adjacent space in the room are highlighted, the boundary conditions are imposed, as well as the direction of gravity is given for calculating the thermogravitational convection of ambient air (fig. 1).
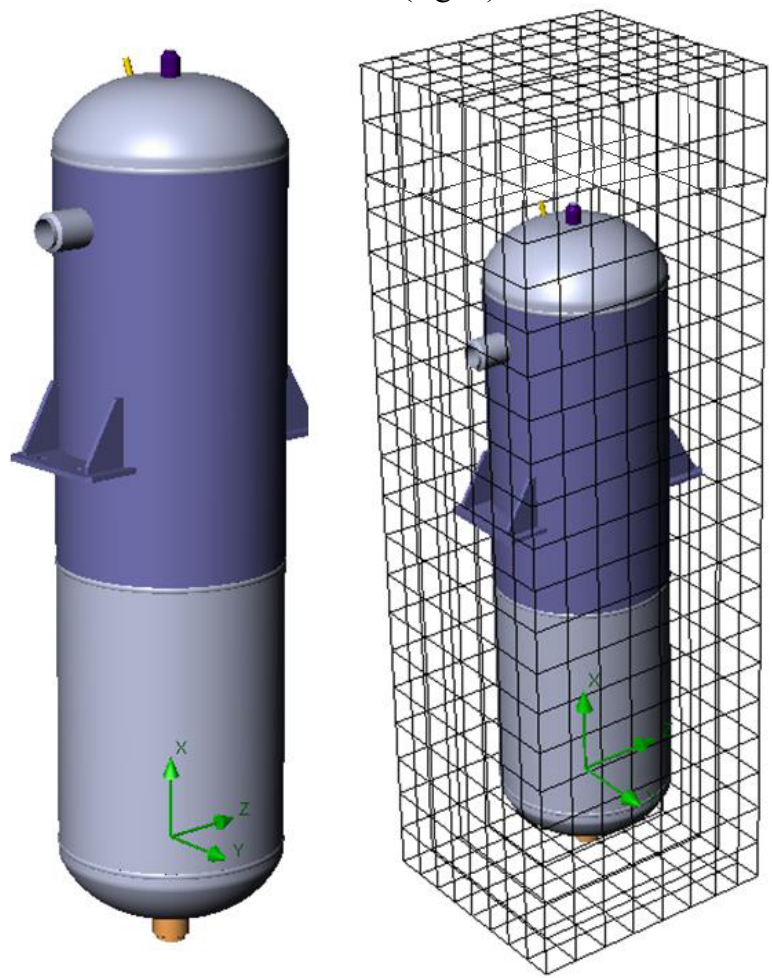

Fig. 1. Three-dimensional structure of the degasser and the basic grid of the model.

Computational grid is built on the basis of computing resources by using its local refinement in the parietal layers, narrow channels and thin walls.

In the modeling, degassers design elements and fluids are assigned to the properties of the respective materials.

The material's thermal conductivity of the degasser's body (which made of stainless steel) in the calculation model was set by using table (Tab. 1) data. 
Table 1. The thermal conductivity of the heat exchange degasser's surface.

\begin{tabular}{|c|c|}
\hline $\begin{array}{c}\text { Temperature, } \\
\mathrm{K}\end{array}$ & $\begin{array}{c}\text { Coefficient of thermal conductivity, } \\
\mathrm{W} /\left(\mathrm{m}^{2} \mathrm{~K}\right)\end{array}$ \\
\hline 293 & 15 \\
\hline 593 & 19.8 \\
\hline 903 & 26.5 \\
\hline 1103 & 27.8 \\
\hline
\end{tabular}

In the path of the inner degasser's surface, which is directly contacting with the circulating water at $348 \mathrm{~K}$, the heat transfer coefficient was taken to be equal $800 \mathrm{~W} /\left(\mathrm{m}^{2} \mathrm{~K}\right)$, and the paths, that are contacting with the gaseous medium $-10 \mathrm{~W} /\left(\mathrm{m}^{2} \mathrm{~K}\right)$. On lattices level there is a mixed contact with the water-gas medium, so the heat transfer coefficient on these surfaces is assumed to be equal $200 \mathrm{~W} /\left(\mathrm{m}^{2} \mathrm{~K}\right)$.

\section{Results of calculation}

Studying of circulating air flows in the surrounded has made it possible to obtain the distribution of the external heat transfer coefficient, heat flux density and temperature of structure elements (fig. 2).
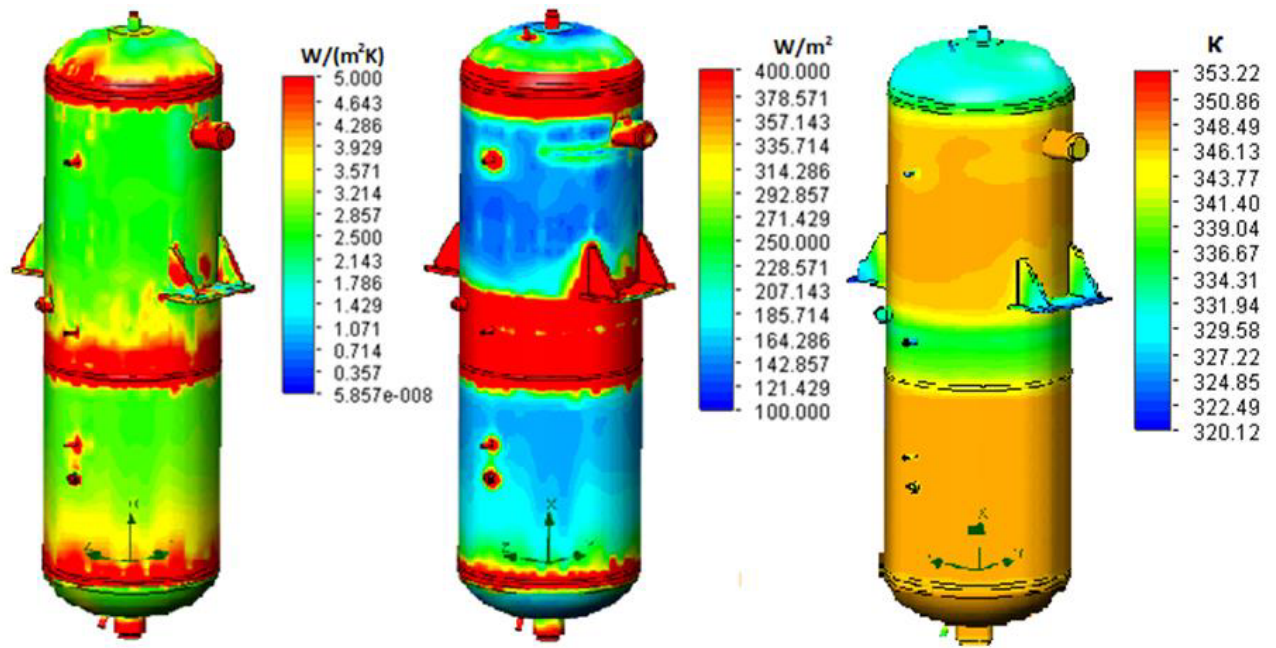

Fig. 2. Estimated distribution of heat transfer coefficient $\left(\mathrm{W} / \mathrm{m}^{2} \mathrm{~K}\right)$, the heat flux $\left(\mathrm{W} / \mathrm{m}^{2}\right)$ and the temperature $(\mathrm{K})$ on the surface of the structural elements.

As a result of the three-dimensional thermal-hydraulic calculation degasser's case, with using the calculating block FLOW SIMULATION packet SolidWorks, was found, that the heat transfer coefficients at the outer surface is unevenly distributed, and changes in the range from 2 to $5 \mathrm{~W} /\left(\mathrm{m}^{2} \mathrm{~K}\right)$, the heat flux may vary from 100 to $400 \mathrm{~W} / \mathrm{m}^{2}$, and the maximum temperature of the base plate's bottom does not exceed $348 \mathrm{~K}$. 


\section{Conclusion}

The general principles laid down in the code and its flexible structure make it possible to mode the heat transfer processes in the units of different configurations and sizes. The proposed approach allows a fast and sufficiently accurate analysis of the efficiency of heat exchange equipment in various operating conditions, including those for mobile small and medium capacity power units.

Using SolidWorks package allows modeling of hydraulic processes in a variety of elements, such as pumps, accumulators, valves of various designs, and so on, which makes it possible to accurately carry out strength calculations.

\section{References}

1. A.G. Bagoutdinova, Y.D. Zolotonosov, O.V. Shemelova, Bulletin of Kazan Technological University 14 (2014)

2. V.A. Ruppel, Yu.S. Tretyakova, S.V. Lavrinenko, et al. SMART GRIDS 2015. MATEC Web Conf. 37, Article Number 01059 (2015)

3. M.S. Khvostova, Secure-ness in the technosphere 2 (2012)

4. V.V. Zamukov, A.E. Baburkin, A.V. Doroshchenko, S.V. Belchenko. Shipbuilding 2 (2007)

5. V.V. Zamukov, A.E. Baburkin, A.V. Doroshchenko, V.S. Stepanov. Probl. At. Sci. Technol., Ser.: Safety Assurance of NPP 24 (2009)

6. A.A. Alyamovsky, A.A. Sobachkin, E.V. Odintsov. Computer modeling in engineering practice (BKHV-Peterburg Publ., St. Petersburg, 2008)

7. K. Larionov, S. Lavrinenko, V. Gubin, et al. XVth International Conference Linguistic and Cultural Studies: Traditions and Innovations (LKTI 2015), Procedia Social and Behavioral Sciences 206 (2015) 